\title{
Modelización térmica de los ríos Cea y Manzanares
}

\author{
Bárbara Gutiérrez \& Diego García de Jalón \\ Laboratorio de Hidrobiología, ETSI de Montes, Universidad Politécnica de Madrid. Avda. Ramiro de Maeztu \\ s/n. 28040 Madrid. España.
}

\section{RESUMEN}

El objetivo de este estudio es analizar las dinámica de las temperaturas de los rios en zonas fluviales de diferentes características: tramos más o menos regulados de dos ríos diferentes de la Península Ibérica, el río Manzanares en su tramo alto y el río Cea en su tramo medio. Tras tomar las temperaturas de estos ríos, cada hora, durante períodos de hasta un año y medio, se han analizado las fluctuaciones térmicas diarias y mensuales. Se han creado dos modelos climáticos fluviales ajustados a los datos obtenidos de manera que definen la pauta que siguen las temperaturas diarias de los rios anteriormente citados y permiten realizar una predicción de estas temperaturas en función a las diferentes características ambientales.

Palabras clave: Temperatura, modelo de crecimiento, modelo sinusoidal.

\begin{abstract}
Temperature dynamics in river sectors with differing degrees of artificial regulation were analysed. Stretches of two Iberian rivers were examined, i.e. the upper River Manzanares and the middle section of the River Cea. Temperature has been measured hourly, adding up to 1.5 years of data. Daily and monthly thermal fluctuations have been analyzed. Two sinusoidal climatic models were developed for these rivers, making possible the prediction of river water temperature as a function of environmental parameters.
\end{abstract}

Keywords: Temperature, growth, sinusoidal model.

\section{INTRODUCCIÓN}

La temperatura es una de las magnitudes físicas que tiene más importancia en el desarrollo de los diversos fenómenos que se realizan en el seno del agua. Determina la evolución o tendencia de sus propiedades, tanto físicas como químicas (Catalán Lafuente \& Catalán Alonso, 1987) o biológicas (Nebeker, 1971; Vannote \& Sweeney, 1980; Ward \& Stanford, 1982; Margalef, 1983; Rader \& Ward, 1990; Brittain, 1991; Brittain \& Campbell, 1991; Salam \& Davies, 1994; García de Jalón, 1995).

Anteriormente, el principal interés del estudio de las temperatura se centraba en los efectos de la deforestación (Moore, 1967; Brown, 1969,1970; Beschta \& Taylor, 1988), la urbanización (Pluhowski, 1970) los vertidos hidroeléctricos e industriales sobre las aguas (Peterson \& Jaske, 1968; Jobson, 1973; Zarik, 1978, Jenkins \& Hansen, 1983) y la construcción de embalses (Webb \& Walling, 1993; Dolz et al., 1995). Sin embargo actualmente la predicción exacta de la temperatura del agua ha adquirido de nuevo gran interés debido a la amenaza de cambio global, y un análisis más dinámico y determinístico de la transferencia de calor en los ríos que se despegue en cierta medida del tradicional análisis de equilibrio de las fuentes de calor es necesario para poder prede- 
cir los efectos de las actividades del hombre en la cuenca de un río (Sinokrot \& Stefan, 1993).

La temperatura de los ríos sigue dos ciclos, uno estacional, y otro diario. A escala estacional, la temperatura del agua depende de la temperatura del aire. La variación diaria de las temperaturas de los ríos está directamente relacionada con los ciclos de radiación solar y temperatura ambiente (Sinokrot \& Stefan, 1993). En el intercambio de calor entre la atmósfera y el río tienen un papel importante parámetros tales como la temperatura del aire, la humedad relativa, la radiación solar, la nubosidad, y la velocidad del viento, siendo estos los parámetros que se emplean en los modelos de suministro de calor (Raphael, 1962; Edinger et al., 1968, 1974; Jobson, 1973; Brocard \& Harleman, 1976). También es muy importante la transmisión de calor a través del lecho del río, especialmente en aquellos de poca profundidad (Brown, 1969; Jobson, 1977; Bowles et al,. 1977). Sin embargo, parece apropiada la creación de un modelo que permita el cálculo de la temperatura de las aguas sin tener en cuenta todos estos parámetros, ya que rara vez se pueden obtener en el campo o, para la zona de estudio en concreto, a partir de las instituciones especializadas.

Por ello, se realiza un análisis de las temperaturas fluviales, de las oscilaciones que muestra la temperatura día a día, proponiendo posteriormente dos modelos sencillos y de fácil empleo, que definen las oscilaciones térmicas.

\section{MATERIAL Y MÉTODOS}

\section{Área de estudio}

La medida de las temperaturas se ha realizado en tres puntos de muestreo de características diferentes. En el río Cea, ya cerca de su desembocadura en el río Esla, a la altura de Melgar de Abajo, al noroeste de la provincia de Valladolid, y en dos puntos del río Manzanares: en su nacimiento, en el arroyo de la Condesa, y en Canto Cochino, también en el tramo alto del río.

\section{Medida de las temperaturas}

En estos puntos se pusieron termómetros de agua que registran temperaturas con precisión de $0,05^{\circ} \mathrm{C}$, cada hora o cada dos horas durante largos períodos de tiempo. Así, para el río Cea se obtuvieron datos de hasta de un año y medio (desde Mayo de 1996 hasta Octubre 1997), y en el río Manzanares de un año (desde Marzo 1997 hasta Marzo 1998).

\section{Análisis de las temperaturas}

Una vez ordenados los datos se ha pretendido definir un modelo que caracterice la fluctuación térmica diaria. Debido a que, independientemente de cuál sea el tipo de río, esta tiene una dinámica oscilatoria (Fig. 1), similar a la que pueda presentar una población con crecimiento limitado y regulado por las diferentes interacciones bióticas o abióticas se ha desarrollado un modelo predictivo en base a la versión de generación discreta del modelo logístico de crecimiento continuo de las poblaciones propuesto por Verhulst (1838) e independientemente por Pearl \& Reed (1920, in Krebs, 1986), ya que cada agrupamiento temporal puede ser diferenciado de los demás (Begon et al., 1995).

El modelo logístico de crecimiento continuo presenta una forma diferencial donde la tasa neta de incremento de una población es $\mathrm{dN} / \mathrm{dt}$, es decir, la velocidad con la que una población aumenta de tamaño, $\mathrm{N}$, a medida que pasa el tiempo, depende del número de individuos de la población, de la tasa de crecimiento per cápita y de lo que Krebs (1986) denomina la "oportunidad no utilizada de crecimiento de la población".

$$
\mathrm{dN} / \mathrm{dt}=\mathrm{Nr}(\mathrm{K}-\mathrm{N} / \mathrm{K})
$$

donde $\mathrm{N}$ es el tamaño de la población, $\mathrm{t}$ el tiempo, $\mathrm{r}$ la tasa de crecimiento per cápita y

$\mathrm{K}$ el valor máximo de $\mathrm{N}$.

A partir de este modelo, para una generación discreta con una tasa constante de multiplicación se obtiene: 
Cea, Junio 96

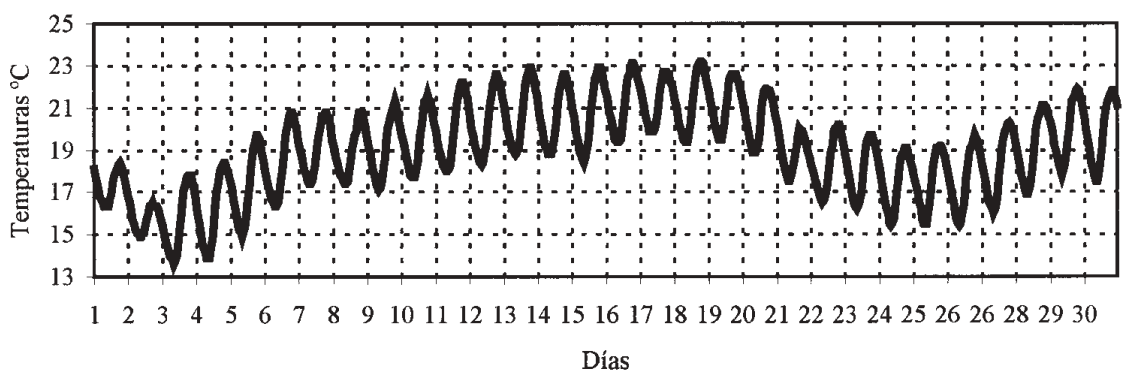

Ayo. Condesa, Abril 97

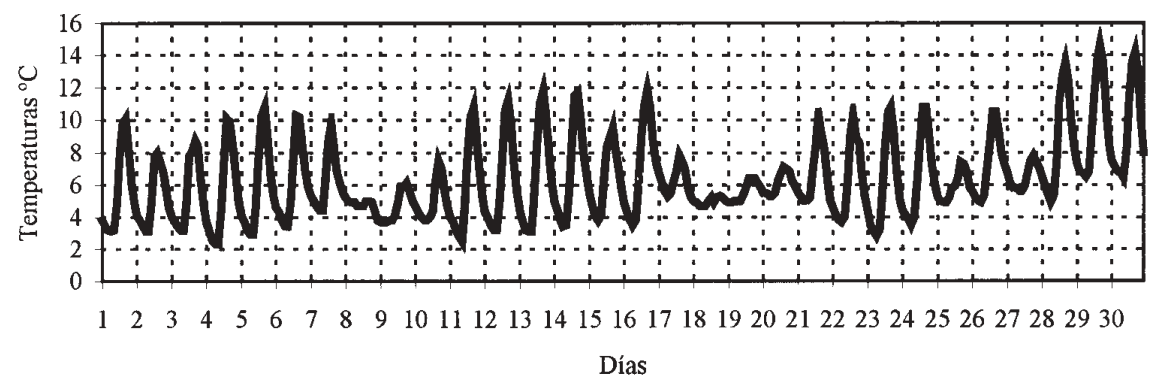

Canto Cochino, Abril 97

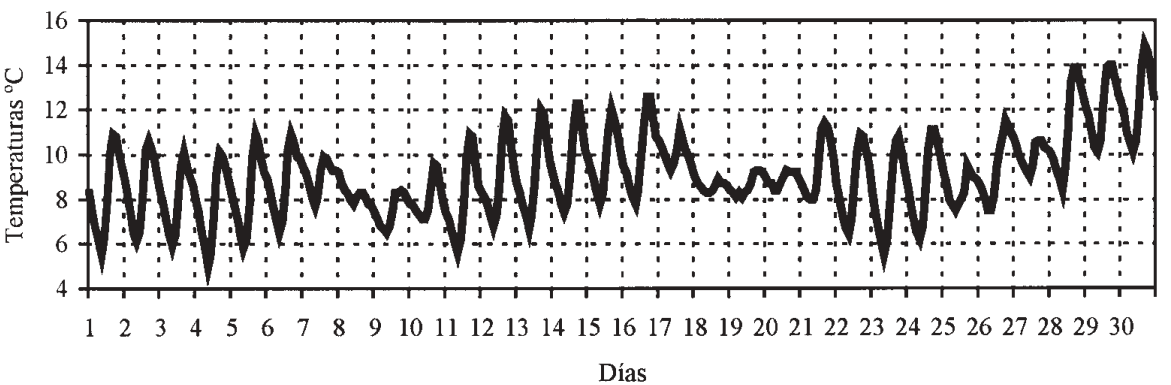

Figura 1. Dinámica oscilatoria de las temperaturas del río Cea, del río Manzanares en el Arroyo de la Condesa y del río Manzanares en Canto Cochino. Oscillatory dynamics of water temperature of River Cea at stream La Condesa, and River Manzanares at Canto Cochino.

$$
\mathrm{N}_{2}=\mathrm{N}_{1} \mathrm{R}
$$

Siendo $\mathrm{N}_{2}$ el tamaño de la población en el tiempo 2, $\mathrm{N}_{1}$ el tamaño de la población en el tiempo 1 y $\mathrm{R}$ la tasa neta de reproducción. Sin embargo, a densidades elevadas las tasas de natalidad decrecen o las de mortalidad aumentan debido a causas diversas como las características del entorno, la disponibilidad de alimento, o las enfermedades. Por tanto, el modelo matemático no puede ser lineal. Habrá un punto de equilibrio en la densidad de la población, $\mathrm{N}_{\mathrm{eq}}$, en torno a la cual se producirán desviaciones de la densidad. Esta desviación de la densidad de equilibrio será:

$$
\mathrm{z}=\mathrm{N}-\mathrm{N}_{\mathrm{eq}}
$$

donde z es la desviación de la densidad de equilibrio, Nel tamaño observado de la población y $\mathrm{N}_{\mathrm{eq}}$ el tamaño de la población en equilibrio. 
Entonces, la tasa reproductiva neta será una línea recta decreciente en función de la densidad. $\mathrm{La}$ ecuación de esta línea es:

$$
\mathrm{R}_{0}=1-\mathrm{B}\left(\mathrm{N}-\mathrm{N}_{\mathrm{eq}}\right)=1-\mathrm{Bz}
$$

En la que B es la pendiente de la recta.

La ecuación 2 de crecimiento puede escribirse entonces de la forma:

$$
\begin{aligned}
& \mathrm{N}_{2}=\mathrm{R}_{0} \mathrm{~N}_{1}=(1-\mathrm{Bz}) \mathrm{N}_{1} \\
& \mathrm{~N}_{2}=\left[1-\mathrm{B}\left(\mathrm{N}-\mathrm{N}_{\mathrm{eq}}\right)\right] \mathrm{N}_{1} \\
& \mathrm{~N}_{2}=\left(1-\mathrm{BN}+\mathrm{BN}_{\mathrm{eq}}\right) \mathrm{N}_{1}
\end{aligned}
$$

El comportamiento de este modelo de población varía en función del valor que adquiera $\mathrm{BN}_{\mathrm{eq}}$, parámetro que se definirá como L. Según sea éste, la población se acerca al equilibrio sin oscilaciones, las oscilaciones son de amplitud decreciente hasta el punto de equilibrio (oscilaciones convergentes), las oscilaciones son cíclicas y estables o la población fluctúa caóticamente de forma más o menos al azar, dependiendo de las condiciones de partida (Maynard Smith, 1968; May, 1974).

Por tanto, se puede considerar a la tempratura del agua como una población cuya densidad $\left({ }^{\circ} \mathrm{C}\right.$ ) varía en cada generación (horas del día), produciendo fluctuaciones diarias (cada 24 generaciones) más o menos estables depen- diendo de factores externos tales como la radiación, el viento, la estación del año, las características ambientales del momento, o el tipo de río y su entorno.

Al igual que en el modelo de crecimiento de las poblaciones, diariamente habrá una tasa de incremento de las temperaturas. Pero estas tasa, en lugar de cambiar con la densidad de la población, varía con lo que en este caso se han denominado generaciones, es decir, horas del día.

Calculando las variaciones de temperatura de una hora a otra se obtenía una gráfica de forma acampanada (Fig. 2), con el máximo entre las doce del medio día y las 16 horas. Dicha gráfica parecía seguir una pauta similar a la que describe una función exponencial cuadrática negativa del tipo:

$$
\mathrm{y}=\mathrm{e}^{-\mathrm{x}^{2}}
$$

por lo que se intentó buscar una función con éstas características que la definiera, pero que a la vez tuviera relación con la función de la tasa de incremento neta de las poblaciones (ecuación 4):

Este modelo, que solo se ha probado con los datos del río Cea, al igual que en el caso de las poblaciones (Maynard Smith, 1974), no pretende representar con exactitud la temperatura en un momento dado, sino, dar una idea de las fluctuaciones diarias y tratar de entender cuales

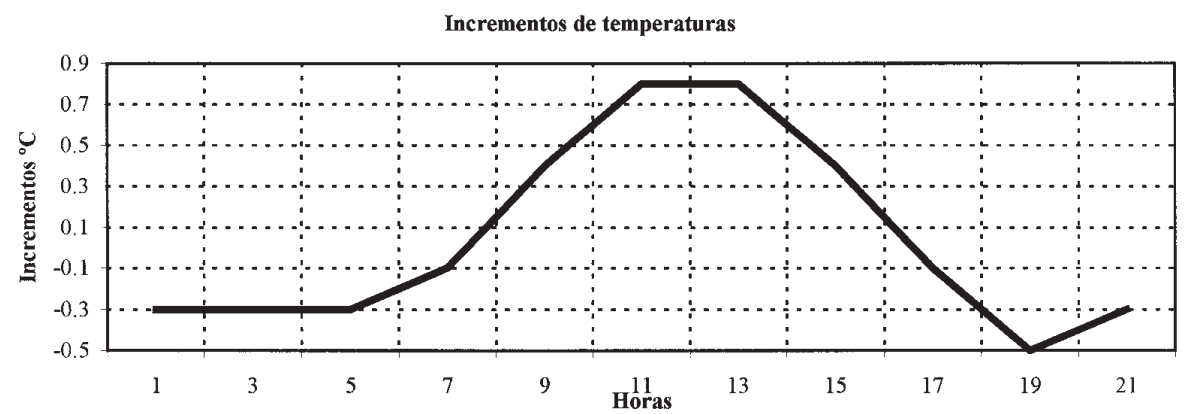

Figura 2. Gráfica de forma acampanada del tipo $\mathrm{y}=\mathrm{e}^{-\mathrm{x}^{2}}$ resultado de las variaciones de temperatura de una hora a otra. Bell-type graph described by the equation $y=e^{-x^{2}}$ giving predicted hourly changes in temperature during one day. 
son los principales factores que determinan las desviaciones de la estabilidad térmica de un río. Para obtener una definición más precisa de las temperaturas diarias se ha creado un segundo modelo:

Sabemos que la temperatura sigue a diario una pauta oscilatoria similar al pulso de una onda, cuya ecuación independiente del espacio (Eisberg \& Lerner, 1984) es:

$$
\mathrm{Y}=\mathrm{A} \operatorname{seno}(2 \mathrm{pt} / \mathrm{T}+\mathrm{d})
$$

A: Es la amplitud de la onda

t: Es cada uno de los momentos en que se define la onda.

T: Es el período de la onda, es decir, el tiempo en que se emplea en realizar un pulso de onda completo.

d: Es la constante de fase, que permite un desplazamiento de la onda a lo largo del eje $\mathrm{x}(\mathrm{t})$. No afecta a la función, pero varía la forma en que se ha definido $t=0$

Anteriormente se han propuesto modelos sinusoidales para las oscilaciones térmicas en las aguas (Hutchinson, 1957; Ward, 1985), pero en estos no se tiene en cuenta que, aunque a primera vista así parezca, la onda de las temperaturas no es perfectamente simétrica (Fig. 3). La longitud de la onda no es igual a lo largo del día. Cuando la temperatura media se mantiene más o menos constante a lo largo de días sucesivos, dicha longitud es mayor durante las mañanas. Esta irregularidad es debido a que la temperatura se eleva rápidamente a medida que lo hace el excedente de radiación. Después del medio día, aunque el excedente de radiación empieza a disminuir, es lo suficientemente grande como para permitir aún un ligero aumento de la temperatura. La máxima temperatura del aire se alcanza entre las 14 y las 16 horas. Esta temperatura del aire también depende de los movimientos turbulentos del aire en las capas inferiores, transportando el aire calentado hacia arriba y sustituyéndolo por aire más frío. El efecto de esta mezcla es hacer que la curva de la temperatura del aire comience a descender mucho antes de que se agote el excedente de radiación. Todo esto ocurre en condiciones equinocciales (21 de Marzo o 23 de Septiembre). Bajo las condiciones del solsticio de junio, se incrementa notablemente la radiación solar incidente. La hora mínima de temperatura se sitúa cerca de las 4 horas, sin embargo, la hora de temperatura máxima se mantiene más o menos constante. En el solsticio de Diciembre tienen lugar las correspondientes reducciones de energía entrante y la hora de temperatura mínima experimenta el correspondiente adelanto (Strahler, 1984).

Para poder corregir las asimetrías de la onda, se han realizado una serie de pruebas con diferentes parámetros matemáticos añadidos a la

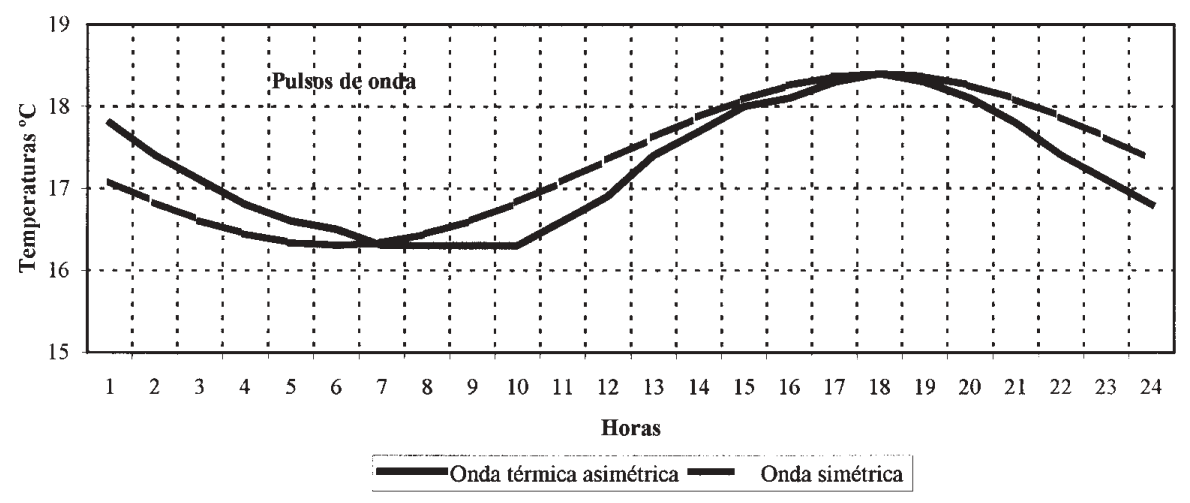

Figura 3. Onda asimétrica que forman las temperaturas a lo largo de un día frente a una onda simétrica. Asymmetric wave formed by temperature changes during one day compared to a symmetric wave. 
ecuación de onda anterior. Tras dichas pruebas, se ha escogido el modelo que mejor se adaptaba a las características térmicas de los ríos muestreados. Este modelo se caracteriza por añadir cuatro exponenciales cuadráticas al seno de la onda, cada una de las cuales corrige un cuarto de la onda.

\section{RESULTADOS}

\section{Modelo basado en el crecimiento de las poblaciones}

En primer lugar, se buscó la función exponencial cuadrática negativa (ecuación 8) que se ajustara a la tasa de incremento de las temperaturas $\left(\Delta \mathrm{T}^{\mathrm{a}}\right)$ a lo largo de un día. De esta forma, se obtiene una función dependiente de la variación térmica diaria y horaria:

$\Delta \mathrm{T}^{\mathrm{a}}=-\mathrm{B}+\mathrm{e}^{-\mathrm{C}(\mathrm{h}-12)^{2}}$

B: es un valor dependiente de la variación térmica que se produce de un día a otro, define la estabilidad térmica entre los días. Este valor disminuye cuando la temperatura media aumenta, y viceversa. Es equiparable al punto de equilibrio, definido en el modelo de crecimiento de las poblaciones. Determina que la oscilación sea convergente, divergente o estable. En una oscilación constante, este valor se encuentra entre 0,31 y 0,32 .

C: ajusta el ancho de la "campana" de la exponencial al del incremento de las temperaturas. h: Es la hora para la que se está calculando el incremento de temperatura. A este dato se le resta 12 para, al igual que en el modelo oscilatorio, definir la posición a lo largo del eje x. Para obtener unos datos más fiables sería más correcto que este valor cambiase según los meses del año, ya que la variación máxima varía entre las 12 y las 15 horas.

Esta función se ajusta al incremento de las temperaturas del río con unos valores que oscilan entre un 70 y un $95 \%$. En la Tabla 1 se exponen los valores de los parámetros de esta fun-

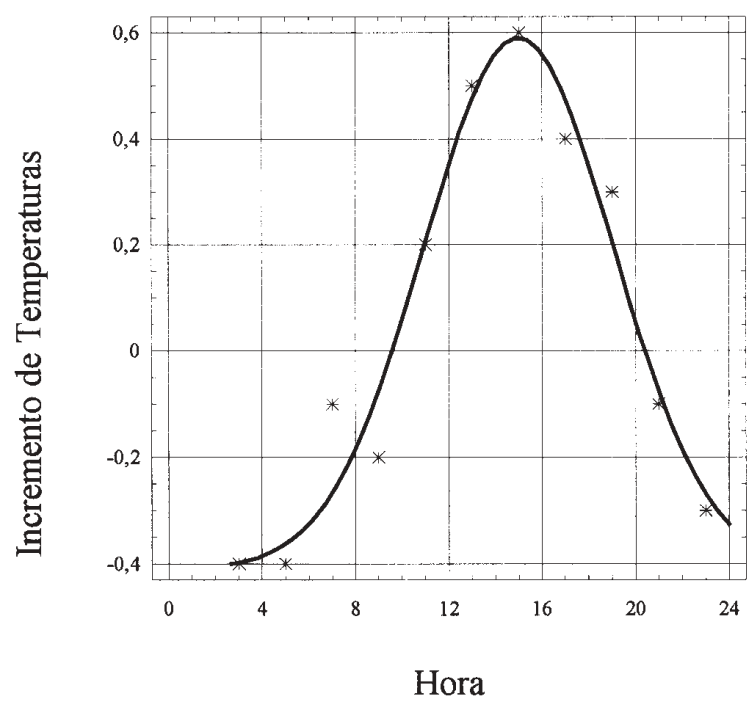

Figura 4. Ajuste de la función exponencial cuadrática negativa a los incrementos de temperatura de un día en el río Cea. Adjustment of the negative quadratic exponential function to temperature increments during one day in the River Cea.

Tabla 1. Ecuación de incremento de temperaturas en diez días a lo largo de un año con los parámetros obtenidos y los diferentes $\mathrm{r}^{2}$. Equation for ten-day temperature increments over one year. Parameters and the $r^{2}$ obtained are also shown.

\begin{tabular}{rcc}
\hline Fecha & Ecuación & $\mathbf{R}^{\mathbf{2}}$ \\
\hline $5-2-97$ & $-0.254+\mathrm{e}^{-0,049(\mathrm{~h}-15)^{2}}$ & 96.32 \\
$1-3-97$ & $-0.281+\mathrm{e}^{-0,0574(\mathrm{~h}-15)^{2}}$ & 86.18 \\
$2-4-97$ & $-0.226+\mathrm{e}^{-0,088(\mathrm{~h}-15)^{2}}$ & 90.16 \\
$25-5-97$ & $-0.234+\mathrm{e}^{-0,087(\mathrm{~h}-13)^{2}}$ & 84.85 \\
$3-6-96$ & $-0.226+\mathrm{e}^{-0,0544(\mathrm{~h}-15)^{2}}$ & 91.00 \\
$1-7-96$ & $-0.307+\mathrm{e}^{-0,0667(\mathrm{~h}-13)^{2}}$ & 92.99 \\
$3-8-96$ & $-0.233+\mathrm{e}^{-0,0796(\mathrm{~h}-14)^{2}}$ & 76.06 \\
$2-9-97$ & $-0.374+\mathrm{e}^{-0,0417(\mathrm{~h}-15)^{2}}$ & 88.45 \\
$1-10-97$ & $-0.355+\mathrm{e}^{-0,057(\mathrm{~h}-13)^{2}}$ & 95.27 \\
$2-10-97$ & $-0.299+\mathrm{e}^{-0,049(\mathrm{~h}-13)^{2}}$ & 92.40 \\
\hline
\end{tabular}

ción para diferentes días a lo largo de todo el año, con los respectivos $r^{2}$. La figura 4 muestran el ajuste de esta ecuación a los incrementos de temperatura de un día en el río Cea. Los días de alta inestabilidad térmica no se ajustan a esta función, ya que las temperaturas no tienen una pauta oscilatoria tan clara, y por tanto los incrementos no se dan de la misma manera. 

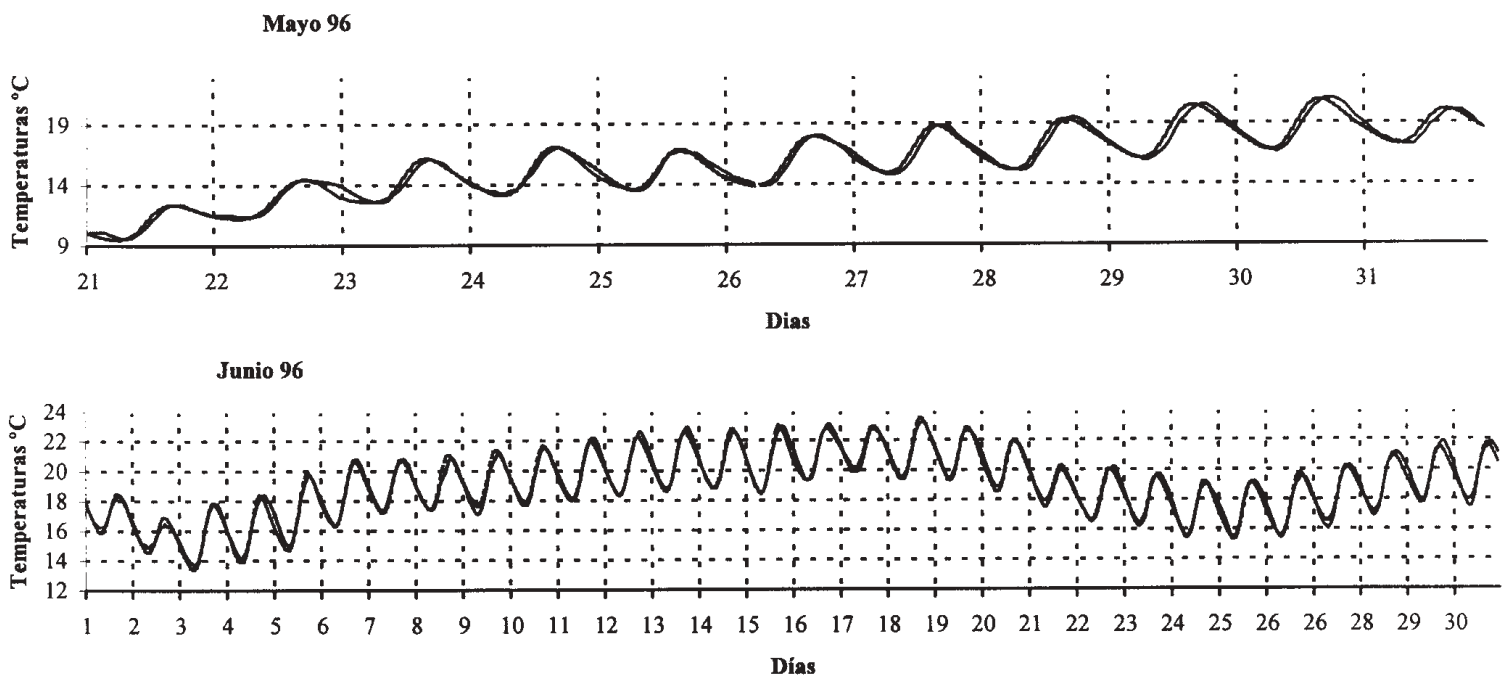

Julio 96

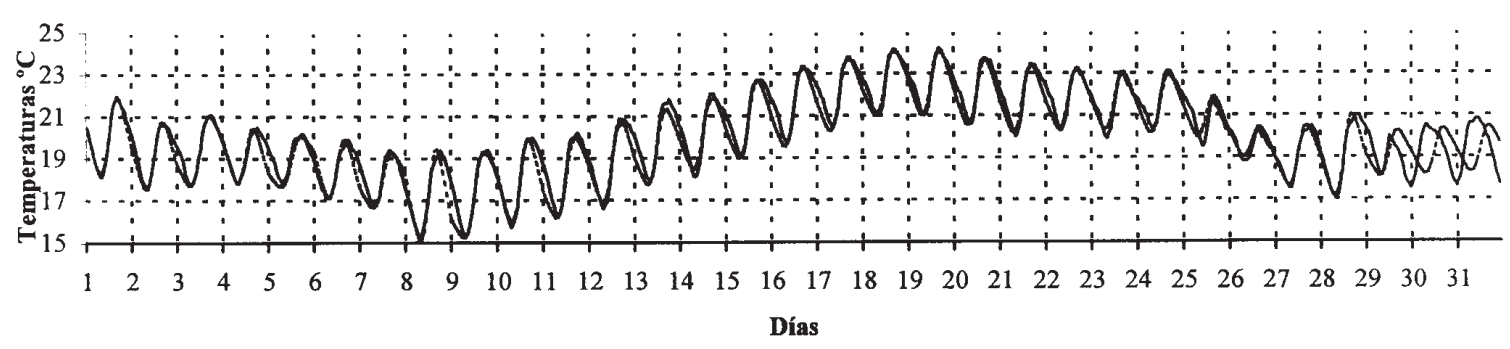

Marzo 97
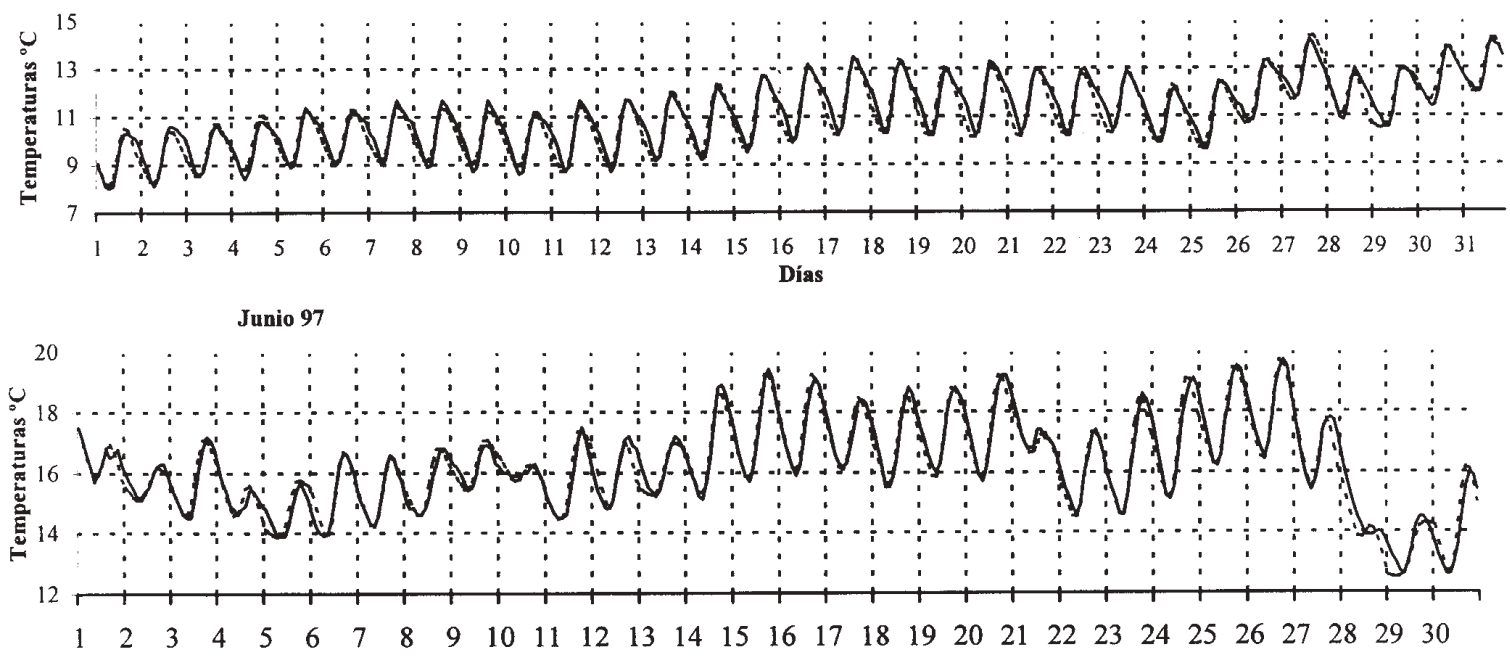

Días

- Datos reales - ... Datos obtenidos

Figura 5. Curva obtenida con el modelo de crecimiento frente a la curva real para los meses de Mayo, Junio y Julio del 1996 y Marzo y Junio 1997. Growth curve compared to plotted observations of temperature for May, June and July, 1996 and March and June, 1997. 
Tomando la ecuación 4 que define la tasa neta de aumento de las densidades de una población y estableciendo una relación con el incremento de las temperaturas tendríamos:

$$
\mathrm{R}_{0}=1+\mathrm{ADT}^{\mathrm{a}}
$$

A: Es un coeficiente que depende, del incremento de las temperaturas con respecto al día anterior, es decir, de la pendiente.

La tasa neta de incremento de las temperaturas a lo largo de un día es por tanto:

$$
\mathrm{R}_{0}=1+\mathrm{A}\left(-\mathrm{B}+\mathrm{e}^{-\mathrm{C}(\mathrm{h}-12)^{2}}\right)
$$

Si tomamos la ecuación 5, y lo aplicamos a las temperaturas tendremos

$$
\mathrm{T}_{2}=\mathrm{T}_{1} \mathrm{R}_{0}
$$

Por lo que finalmente tendríamos

$$
\mathrm{T}_{2}=\mathrm{T}_{1}\left[1+\mathrm{A}\left(-\mathrm{B}+\mathrm{e}^{-\mathrm{C}(\mathrm{h}-12)^{2}}\right)\right]
$$

Diversos estudios (Macan, 1959; Edington, 1965; Wetzel, 1981; Crisp \& Houston, 1982; Ward, 1985) han demostrado que las temperaturas de los ríos, especialmente en los poco profundos, varían linealmente en función de la temperatura ambiental y están estrechamente relacionados con las características atmosféricas de la zona. Siendo las regresiones diferentes para cada tipo de río. Por tanto, si sabemos la regresión que define la relación de la temperatura ambiental con la de un río, y sabemos las características térmicas ambientales para un período de tiempo definido, podremos establecer el régimen térmico del río en dicho período teniendo una única medida de temperatura de sus aguas, ya que gracias a esta medida podremos obtener la temperatura a las hora siguiente, y con ésta, la temperatura siguiente, y así sucesivamente.

La figura 5 muestra el funcionamiento de esta función para los meses de Mayo, Junio y Julio del 96 y Marzo y Junio del 97. En estos ejemplos, para facilitar el cálculo se ha empleado un valor constante de $\mathrm{C}$ de 0.0535 . También
Tabla 2. $\mathrm{R}^{2}$ con los que se ajusta la función basada en el incremento de las poblaciones para los meses de Marzo-97, Mayo-96, Junio 96 y 97 y Julio 96. $R^{2}$ for the adjustment of the function based on the increment of populations for March 1997, May 1996, June 1996, June 1997 and July 1996.

\begin{tabular}{ll}
\hline Fecha & $\mathbf{R}^{\mathbf{2}}$ \\
\hline Mayo 96 & 0.99 \\
Junio 96 & 0.97 \\
Julio 96 & 0.91 \\
Marzo 97 & 0.93 \\
Junio 97 & 0.95
\end{tabular}

se ha tomado como constante la hora a la cual el incremento máximo de temperatura tiene lugar, siendo ésta a las 12 del medio día. En la Tabla 2 se muestran los $\mathrm{R}^{2}$ con los que se ajusta la función para estos ejemplos.

Como se ha dicho antes, el valor de los coeficientes A y B no solo depende del incremento de la temperatura, también dependen de la media diaria, es decir, a incrementos de temperatura iguales, no tienen el mismo valor cuando la temperatura media es $5^{\circ} \mathrm{C}$ que cuando ésta es de $15^{\circ} \mathrm{C}$.

\section{Modelo sinusoidal con cuatro exponenciales}

Como una función sinusoidal oscila en torno al valor medio 0 , con valores positivos y negativos, al ajustar la ecuación de onda expuesta anteriormente (ecuación 9) a las temperaturas diarias tendríamos que sumarle el valor medio de la temperatura, es decir,

$$
\mathrm{B}=\left(\mathrm{T}^{\mathrm{a}} \max +\mathrm{T}^{\mathrm{a}} \min \right) / 2
$$

La amplitud A, la da el valor de la diferencia entre la temperatura máxima y la mínima dividido por dos:

$$
\mathrm{A}=\left(\mathrm{T}^{\mathrm{a}} \max -\mathrm{T}^{\mathrm{a}} \min \right) / 2
$$

t: es cada una de las 24 horas del día, por lo que lo denominaremos $\mathrm{h}$.

$\mathrm{T}$ : es el período o tiempo en que tiene lugar un pulso de onda completo, es decir, 24 horas.

d: define la posición de la onda a lo largo del eje $\mathrm{x}(\mathrm{h})$. Como el valor medio de las tempera- 
turas de un día se da aproximadamente a las 12 del medio día, con variaciones según las épocas, este valor es de aproximadamente $0.85 \mathrm{p}$. Pero, otra manera de definir la posición de la ecuación a lo largo del eje es restando el valor de $t$ en el momento en que tiene lugar la temperatura media del día (aproximadamente las 12) al parámetro t de la ecuación.

Por tanto la ecuación que define las temperaturas para un día según el modelo oscilatorio sería:

$$
\begin{aligned}
& \mathrm{Y}=\mathrm{B}+\mathrm{A} \text { seno }(2 \mathrm{p}(\mathrm{h}-12) / 24) \\
& \text { (ecuación 17) }
\end{aligned}
$$

Como se dijo anteriormente, este modelo define la oscilación térmica diarias de un río de una manera más precisa, con una función sinusoidal corregida debido a sus asimetrías. Tras una serie de pruebas, esto se ha conseguido sumándole a dicha función sinusoidal cuatro exponenciales cuadradas, cada una de las cuales corrige un cuarto de la onda, estas exponenciales las denominaremos $\mathrm{a}, \mathrm{b}, \mathrm{g}$ y $\mathrm{m}$. Por tanto la ecuación quedaría de la siguiente manera:

$$
\begin{aligned}
& \mathrm{T}^{\mathrm{a}}=\mathrm{B}+\mathrm{A} \text { seno[}[(2 \mathrm{p} / 24)(\mathrm{h}-12+\mathrm{a}+\mathrm{b}+\mathrm{g}+\mathrm{m})] \\
& (18) \\
& \text { a: Corrige el primer cuarto de la ecuación } \\
& \mathrm{a}=a \mathrm{e}^{\left(-0,05 \mathrm{~h}^{2}\right)} \\
& \mathrm{b}: \text { Corrige el segundo cuarto de la ecuación } \\
& \mathrm{b}=b \mathrm{e}^{\left(-0,05(\mathrm{~h}-8)^{2}\right)} \\
& \mathrm{g}: \text { Corrige el tercer cuarto de la ecuación } \\
& \mathrm{g}=c \mathrm{e}^{\left(-0,05(\mathrm{~h}-16)^{2}\right)} \\
& \mathrm{m}: \text { Corrige el cuarto cuarto de la ecuación } \\
& \mathrm{m}=d \mathrm{e}^{\left(-0,05(\mathrm{~h}-24)^{2}\right)}
\end{aligned}
$$

$a, b, c$ y $d$ son coeficientes que dependen de las características ambientales del día

Por tanto, la ecuación final quedaría de la siguiente manera:

\footnotetext{
$\mathrm{T}^{\mathrm{a}}=\left(\mathrm{T}^{\mathrm{a}} \max +\mathrm{T}^{\mathrm{a}} \min \right) / 2+\left[\left(\mathrm{T}^{\mathrm{a}} \max -\mathrm{T}^{\mathrm{a}} \min \right) / 2\right]$ seno $\left[(2 \mathrm{p} / 24)\left(\mathrm{h}-12+a \mathrm{e}^{\left(-0.05 \mathrm{~h}^{2}\right)}+b \mathrm{e}^{\left(-0.05(\mathrm{~h}-8)^{2}\right)}+c \mathrm{e}^{(-}\right.\right.$ $\left.\left.0.05(\mathrm{~h}-16)^{2}\right)+d \mathrm{e}^{\left(-0.05(\mathrm{~h}-24)^{2}\right)}\right]$

Esto es así cuando el cálculo del seno se hace en radianes. En el caso de que se calcule en grados hay que multiplicar por $180 / \mathrm{p}$ por lo que la ecuación quedaría de la forma:

$$
\begin{gathered}
\mathrm{T}^{\mathrm{a}}=\left(\mathrm{T}^{\mathrm{a}} \max +\mathrm{T}^{\mathrm{a}} \min \right) / 2+\left[\left(\mathrm{T}^{\mathrm{a}} \max -\right.\right. \\
\left.\left.\mathrm{T}^{\mathrm{a}} \min \right) / 2\right] \text { seno }\left[1 5 \left(\mathrm{h}-12+a \mathrm{e}^{\left(-0.05 \mathrm{~h}^{2}\right)}+b \mathrm{e}^{(-0.05(\mathrm{~h}-}\right.\right. \\
\left.\left.8)^{2}\right)+c \mathrm{e}^{\left(-0.05(\mathrm{~h}-16)^{2}\right)}+d \mathrm{e}^{\left(-0.05(\mathrm{~h}-24)^{2}\right)}\right]
\end{gathered}
$$

Esta función explica la oscilación diaria de las temperaturas con valores de $\mathrm{R}^{2}$ entre un $90 \mathrm{y}$ un $99 \%$, a excepción de aquellos días en los que la inestabilidad térmica es tan grande que la temperatura no sigue una pauta oscilatoria. La figura 6 muestra el ajuste entre los datos obtenidos en el campo y los datos obtenidos con el modelo para un día de invierno y otro de verano en el río Cea y para los dos puntos del río Manzanares en Marzo. En la Tabla 3 se muestran valores de los diferentes parámetros para esta ecuación en dichos ejemplos.

\section{CONCLUSIÓN}

A la hora de crear un modelo capaz de predecir las fluctuaciones térmicas en los ríos deben tenerse en cuenta todo tipo de interacciones y perturbaciones, desde el origen de las aguas, las características ambientales hasta los usos de la cuenca vertiente. Para llegar a unas condiciones fiables, es necesaria la cooperación entre diferentes disciplinas y, por supuesto, estudios de las características térmicas de los diferentes tipos de ríos. De momento, debido a la complejidad de los sistemas climáticos, a la falta de un conocimiento completo de todos los procesos que actúan así como a la ausencia de los datos necesarios para la puesta en marcha de los modelos de balance de energía, los modelos térmicos de los ríos han de limitarse a ser una simplificación del mundo real. Estas simplificaciones son de dos tipos: Una que afecta a los propios procesos, que como se acaba de decir es generalmente debido a la limitación de la comprensión de estos o a la ausencia de datos, y la segunda, que implica la resolución del modelo 
Tabla 3. Parámetros para la ecuación sinusoidal y $\mathrm{r}^{2}$ con los que se ajustan en un día de invierno y otro de verano en el río Cea y para los dos puntos del río Manzanares en Marzo. Parameters for the sinusoidal equation and $r^{2}$ obtained for the adjustment of one winter day and one summer day in the River Cea and at two locations of the River Manzanares.

\begin{tabular}{cccccccc}
\hline & B & A & $\boldsymbol{a}$ & $\boldsymbol{b}$ & $\boldsymbol{c}$ & $\boldsymbol{d}$ & $\mathbf{R}^{\mathbf{2}}$ \\
\hline $\begin{array}{c}\text { Cea } \\
\mathbf{2 8 - 2 - 9 7} \\
\mathbf{C e a}\end{array}$ & 8.95 & 0.95 & 0.7719 & -0.7744 & 1.6625 & 0.0447 & 99.78 \\
$\begin{array}{c}\mathbf{6 0 - 6 - 9 7} \\
\text { Ayo. Condesa } \\
\mathbf{3 0 - 3 - 9 7}\end{array}$ & 14.30 & 1.70 & 1.8415 & -1.5105 & -1.2822 & -0.5588 & 99.75 \\
$\begin{array}{c}\text { Canto Cochino } \\
\mathbf{2 6 - 3 - 9 7}\end{array}$ & 6.45 & 3.34 & 2.0890 & -0.5870 & 4.5460 & 4.6310 & 99.18 \\
\hline
\end{tabular}

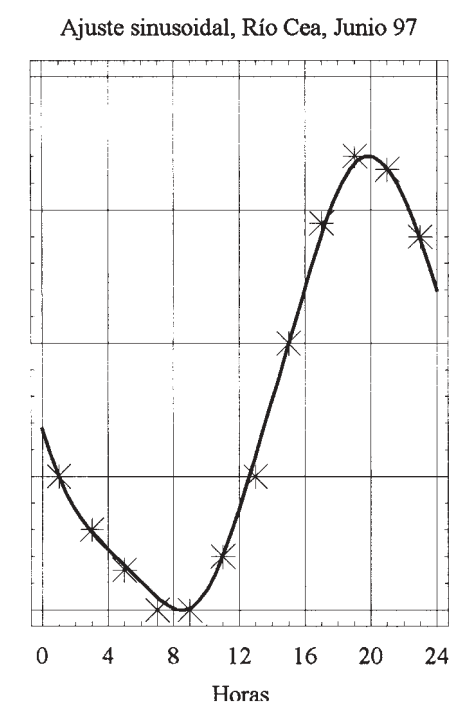

Ajuste Sinusoidal, Rio Cea, Febrero 97

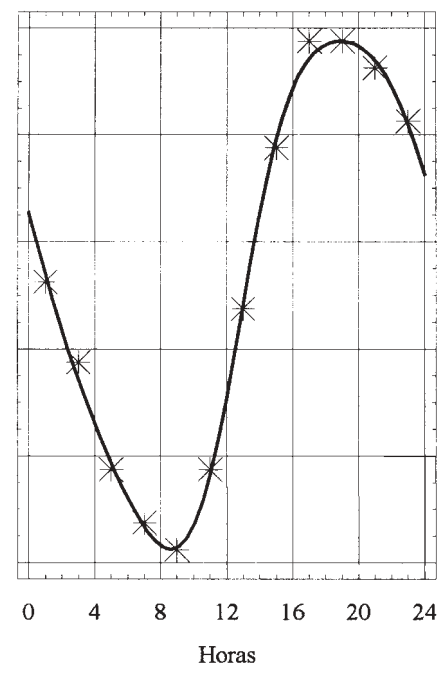

Ajuste Sinusoidal, Ayo. Condesa, Marzo 97

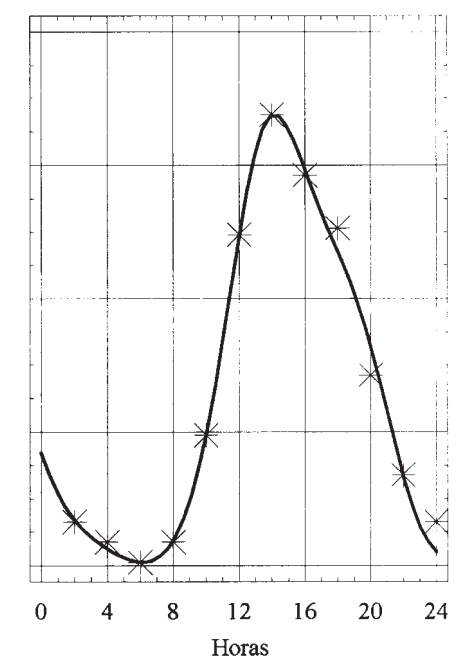

Ajuste Sinusoidal, Canto Cochino, Marzo 97

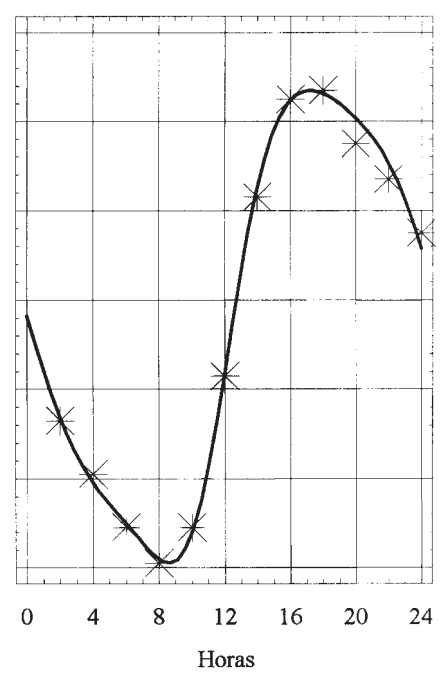

Figura 6. Ajuste del modelo sinusoidal a los datos de campo para un día de verano y otro de invierno del río Cea y para los dos puntos del río Manzanares en Marzo. Adjustment of the sinusoidal model to field data for one summer day and for one winter day for River Cea and for the two sites of River Manzanares in March 1997.

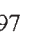


tanto en el tiempo como en el espacio, pues aunque generalmente cuanto mejor sea la resolución, mejor serán los resultados, una resolución excesivamente buena podría ser inapropiada debido a que los procesos que actúen a escalas menores que el modelo destinado a explicarlos podrían ser incorporados inadvertidamente (Henderson-Sellers \& McGuffie, 1990). Además, por muy bueno que sea un modelo predictivo, siempre tendrá el defecto de que su funcionamiento sólo puede ser comprobado con las temperaturas del pasado de las que existan datos y con las actuales.

Tras la obtención de los modelos expuestos anteriormente, es necesario un estudio preciso de los proceso que interactúan en el clima de las zonas analizadas (situación geográfica, vientos, polución, regulación del caudal mediante embalses) al igual que todos aquellos que afectan en mayor o menor medida a las características físico químicas de las aguas y de la cuenca vertiente, para poder así definir empíricamente los coeficientes que ajustan el modelo a las temperaturas reales, intentando que esta "submodelización" mantenga una sencillez a la hora de su empleo a la vez que mantiene un equilibrio entre una precisión relativamente buena a escala local y un funcionamiento a escala más global, o por lo menos regional.

\section{BIBLIOGRAFÍA}

BEGON, M., J. L. HARPER \& C. R. TOWNSEND. 1995. Ecología. Barcelona: Omega.

BESCHTA, R. L. \& R. L. TAYLOR. 1988. Stream temperature increases and land use in a forested Oregon watershed. Water Resources Bulletin. 24 (1): 19-25.

BOWLES, S. B., D. L. FREAD \& W. J. GRENNEY. 1977. Coupled dynamic streamflow-temperature models. J. Hydraul. Div. Am. Soc. Civ. Eng., 102: 227-240.

BRITTAIN, J. E. 1991. Effect of temperature on egg development in the Australian stonefly genus Austrocercella (Plecoptera: Notonemuridae). Aus. J. Mar. Freshwater Res., 42: 107-114.
BRITTAIN, J. E. \& I. C. CAMPBELL. 1991. The effect of temperature on egg development in the Australian mayfly genus Coloburiscoides (Ephemeroptera: Coloburiscidae) and its relationship to distribution and life history. Journal of Biogeography, 18: 231-235.

BROCARD, D.N. \& D. R. F. HARLEMAN. 1976. One dimensional temperature predictions in unsteady flow. J. Hydraul. Div. Am. Soc. Civ. Eng., 102: 227-240.

BROWN, G.W. 1969. Predicting temperatures of small streams. Water Resour. Res., 5(1): 68-75.

BROWN, G.W. 1970. Predicting the effect of clearcutting on stream temperature. J. Soil Water Conservation, 25: 11-13.

CATALÁN LAFUENTE, J. \& J. M. CATALÁN ALONSO. 1987. Ríos, caracterización y calidad de sus aguas. Madrid: Bellisco.

CRISP, D. T. \& G. HOWSON. 1982. Effect of air temperature upon mean water temperature in streams in the north Pennines and English Lake District. Freshwater Biology, 12: 359-367.

DOLZ, J., J. PUERTAS \& E. HERRERO. 1995. Incidencia de los embalses en el comportamiento térmico de un río. In: Efectos térmicos en presas y embalses. J. Dolz, J. Puertas, A. Aguado \& L. Agulló (eds.): 9-26. Colegio de Ingenieros de Caminos, Canales y Puertos. Madrid.

EDINGER, J. E., D. W. DUTTWEILER \& J. C. GEYER. 1968. The response of water temperatures to meteorological conditions. Water Resour. Res. 4(5) 1137-1143.

EDINGER, J. E., D. K. BRADY \& J. C. GEYER. 1974. Heat exchange and transport in the environment, Rep. 14. Electr. Power Res. Ins., Palo Alto, Calif.

EDINGTON, J. M. 1965. Some observations on stream temperature. Oikos, 15: 265-273.

EISBERG, R. M. \& L. S. LERNER. 1984. Física: Fundamentos y Aplicaciones. Vol. I. México: McGraw-Hill.

GARCÍA DE JALÓN, D. 1995. Impactos de las modificaciones del régimen térmico en las comunidades fluviales. In: Efectos térmicos en presas y embalses J. Dolz, J. Puertas, A. Aguado \& L. Agulló (eds.): 95-108. Colegio de Ingenieros de Caminos, Canales y Puertos. Madrid.

HENDERSON-SELLERS, A. \& K. McGUFFIE. 1990. Introducción a los modelos climáticos. Barcelona: Omega. 
HUTCHINSON, G. E. 1957. A Treatise on Limnology, Vol I: Geography, Physics and Chemistry. New York: John Wiley \& Sons.

JENKINS, S. H. \& P.S. HANSEN. 1983. Cooling water discharges from coal fired power plants - Water pollution problems. Water Sci. Technol. 15 (10).

JOBSON, H. E. 1973. The dissipation of excess heat from water systems. J. Power Div. Am. Soc. Civ. Eng., 99: 89-103.

JOBSON, H. E. 1977. Bed conduction computations for thermal models. J. Hydraul. Div. Am. Soc. Civ. Eng., 103(HY10): 1213-1216.

KREBS, C. J. 1986. Ecología. Madrid: Pirámide.

MACAN, T. T. 1959 The temperature of a small stony stream. Freshwater Biological Association, Ambleside, Westmorland, England.

MARGALEF, R. 1983. Limnología. Barcelona. Omega.

MAY, R. M. 1974. Biological populations with nonoverlapping generations: stable points, stable cycles and chaos. Science, 186: 645-687.

MAYNARD SMITH, J. 1968. Mathematical Ideas in Biology. New York: Cambridge University Press.

MAYNARD SMITH, J. 1974. Models in Ecology. New York. Cambridge University Press.

MOORE, A. M. 1967. Correlation and analysis of water temperature data for Oregon streams. U.S. Geol. Surv. Water Supply Pap., 1819-K.

NEBEKER, A.V. 1971. Effect of high winter water temperatures on adult emergence of aquatic insects. Water Research 5: 777-783.

PEARL, R. \& L. J. REED. 1920. On the rate of growth of the population of the United States since 1790 and its mathematical representation. Proc. Natl. Acad. Sci. USA, 6: 275-288. Citado en Krebs, C. J., 1986. Ecología. Madrid. Pirámide.

PETERSON, D. E. \& R. T. JASKE. 1968. A test simulation of potential effects of thermal power plants on streams on the upper Mississippi River basin, Batelle Northwest laboratories. Rep. BNWL-999, UC-2. Batelle Northwest lab., Richland, Washington.
PLUHOWSKI, E.J. 1970. Urbanization and its effects on the temperature of streams on Long Island, New York. U. S. Geol. Surv. Prof. Pap., 627-D.

RADER, R. B. \& J. V. WARD. 1990. Mayfly growth and population density in constant and variable temperature regimes. The Great Basin Naturalist 50 (2): 97-106

RAPHAEL, J. M. 1962. Prediction of temperature in rivers and reservoirs. J. Power Div. Am. Soc. Civ. Eng., 88(PO2): 157-181.

SALAM, A. \& P. M. C. DAVIES. 1994. Effect of body weight and temperature on the maximum daily food consumption of Esox lucius. Journal of Fish Biology, 44: 165-167

SINOKROT B. A. \& H. STEFAN. 1993. Stream temperature dynamics: Measurements and modelling. Water Resources Research, 29 (7): 2299-2312.

STRAHLER, A.N. 1984. Geografía Física. Barcelona: Omega.

VANNOTE, R. L. \& B. W. SWEENEY. 1980. Geographic analysis of thermal equilibria: a conceptual model for evaluating the effect of natural and modified thermal regimes on aquatic insects communities. American Naturalist, 115: 667-695.

VERHULST, P. F. 1838. Notice sur la loi que la population suit dans son accroissement. Corresp. Math. Phys., 10: 113-121. Citado en Krebs, C. J., 1986. Ecología. Madrid: Pirámide.

WARD, J. V. 1985. Thermal characteristics of running waters. Hydrobiologia, 125: 31-46.

WARD J. V. \& J. A. STANFORD. 1982. Thermal responses in the evolutionary ecology of aquatic insects. Ann. Rev. Entomol., 27: 97-117.

WEBB, B. W. \& D. E. WALLING. 1993. Temporal variability in the impact of river regulation on thermal regime and some biological implications. Freshwater Biology, 29: 167-182.

WETZEL, R. G. 1981. Limnología. Barcelona: Omega.

ZARIC, Z. P. (ed.) 1978: Thermal Effluent Disposal From Power Generation. New York: Hemisphere. 